\title{
Numerical Study on the Anti-Snow Performance of Deflectors on a High-Speed Train Bogie Frame
}

\author{
G. Gao, Q. Chen, J. Zhang, Y. Zhang, Z. Tian and C. Jiang ${ }^{\dagger}$ \\ Key Laboratory of Traffic Safety on Track of Ministry of Education, School of Traffic \& Transportation \\ Engineering, Central South University, Changsha, Hunan, 410075, China \\ National \& Local Joint Engineering Research Center, Safety Technology for Rail Vehicle, Changsha, Hunan, \\ 410075, China \\ Joint International Research Laboratory of Key Technology for Rail Traffic Safety, Central South University, \\ Changsha 410075, China \\ † Corresponding Author Email: jiangchen2007@hotmail.com
}

(Received October 21, 2019; accepted February 24, 2020)

\begin{abstract}
In this paper, a new installation of flat plate deflector which attached on the bottom of the bogie frame is proposed and its anti-snow accumulation performance with different attack angles is numerically studied. The wind-snow two-phase flow in the bogie region is simulated based on the Reynolds Averaged Navier-Stokes (RANS) equations combined with the Realizable $k-\varepsilon$ turbulence model and the Lagrangian particle phase method. The adopted numerical simulation methodology is verified and validated by comparing with previous wind tunnel tests. In this paper, three typical attack angles $\left(30^{\circ}, 60^{\circ}, 90^{\circ}\right)$ for deflector are studied. The results show that: the $30^{\circ}$ case has a medium influence on the flow field and reduces snow accumulation by $35.14 \%$; the $60^{\circ}$ case guides the high-speed airflow downward and has the best effect with $62.46 \%$ reduction in snow accumulation; the $90^{\circ}$ case has the smallest reduction with $20.30 \%$ in the mass. Overall, all deflectors with three different installation angles can reduce the mass of snow accumulated on the bogie surface.
\end{abstract}

Keywords: Snow accumulation; Lagrangian particle phase; Wind-snow two-phase flow; High-speed train bogie.

\section{NOMENCLATURE}

$A_{p} \quad$ projected region of the particle

$C_{d} \quad$ drag coefficient of the particle

$\boldsymbol{F}_{d} \quad$ drag force

$\boldsymbol{F}_{g} \quad$ gravity force

$\boldsymbol{F}_{L S} \quad$ shear lift force

$\boldsymbol{F}_{p} \quad$ pressure gradient force

$\boldsymbol{f}_{\boldsymbol{u}} \quad$ user body force (per unit volume).

$\boldsymbol{F}_{u} \quad$ user-defined body force

$\boldsymbol{F}_{v m} \quad$ virtual mass force

$P_{\infty} \quad$ far field pressure and set it to 0 .

$u_{p x} \quad$ velocity of particle in the $x$ direction

$U_{\text {ref }} \quad$ inflow velocity

$u_{x} \quad$ time average velocity of $\mathrm{x}$ direction

\section{INTRODUCE}

In the high-speed train operation in cold area, the snow on the ground will whirl around the high-speed train because of the airflow induced by train passage

$\begin{array}{ll}\boldsymbol{v} & \begin{array}{l}\text { instantaneous velocity of the continuous } \\ \text { phase }\end{array} \\ \boldsymbol{v}_{p} & \text { velocity of particle } \\ V_{P} & \text { volume of the particle } \\ \boldsymbol{v}_{s} & \text { particle slip velocity } \\ \boldsymbol{g} & \text { gravitational acceleration vector } \\ H & \text { height of the high-speed train model } \\ P & \text { time average pressure } \\ \boldsymbol{\omega} & \text { curl of the fluid velocity } \\ \nabla p_{\text {static }} & \text { gradient of the static pressure in the } \\ & \text { continuous phase }\end{array}$

(Kloow 2006). The snow particles will be sucked into the bogie region due to the differential pressure caused by the complex structure of the bogie (Kloow 2006). Once snow particles adhered to the bogie surface, the snow will become more and more 
serious. (Xie et al. 2017) However, bogie is an important part of high-speed train running gear related to the braking and running comfort. The accumulated snow can deteriorate performance of brake clamp, normal movement of air spring, heat dissipation of motor and normal operation of gear box (Kloow 2006, Bettez 2011). If a large amount of ice and snow seriously accumulated in the bogie region, train running safety is severely endangered (Kloow 2006, Fujii et al. 2002).

At present, two main solutions are widely used to relieve the problem of limited high-speed train operation in winter. One way is preventing or reducing snow accumulation in bogie region. Another is de-icing the bogie region (Wiebe et al. 2010). Since the snow particles carried by airflow entering bogie region are redirected or controlled, the de-icing approach is unnecessary. Brenna et al. (2007) proposed that a downward inclined guide plate could be added under the driver's cab brush to prevent the rising airflow and avoid the snow particles from blocking the driver's sight. Wiebe et al. (2010) proposed deflectors added at the front of the bogie to prevent snow packing. However, the effectiveness of above optimized schemes is not verified.

In recent years, some scholars have carried out several numerical studies on the wind-snow twophase flow in the region of high-speed train bogie to propose potential schemes to prevent snow accumulation. Paradot et al. (2014) used the DPM provided by ANSYS Fluent to simulate the snow accumulation of trains in snowstorm environment. $\mathrm{Wu}$ (2015) studied the external flow field around the high-speed train carriage using RANS combined with realizable $k-\varepsilon$ model and DPM. Gao et al. (2018a) conducted numerical simulation of windsnow two-phase flow by using RNG two-equations turbulence model and DPM, and validated the numerical simulation method through wind tunnel experiments. It is confirmed that the high-speed airflow will go upward into the bogie region, leading the snow particles carried in the airflow enter the bogie region. In addition, they also proposed the diversions front end and rear most end of the bogie region and explored the anti-snow performance of the diversions. Xie et al. (2017) studied the characteristics of flow field and snow covering around the bogie region, and verified the numerical simulation through experiments. By analyzing the similarities and differences of snow distribution on bogie surface under different working conditions, the performance of deflectors installed at front and rear ends of bogie cavity was obtained. Gao et al. (2018b) studied the distribution characteristics of snow accumulation on the bogie surface of high-speed train under the condition of crosswinds, and studied the influence of fairing height on the amount of snow particles in each component. Liu et al. (2018) used the IDDES and DPM discrete phase to simulate the train running in snowy environment. Besides, the characteristics of particles in the bogie region and the mass of particles on different parts were studied. Wang et al. (2018b) numerically and experimentally studied the snow distribution characteristics of a bogie model with different angles of the deflector installed on the both sides of the train. Wang et al. (2019a) designed the snow shields with three types for brake calipers and analyzed the anti-snow effects of the devices. Wang et al. (2019b) also discussed the influence of the length of bogie cavities and the fairing shape on snow accumulation in bogie region, which provided ideas for prevention and treatment of snow ice in bogie region. Cai et al. (2018) studied the flow field and particle motion characteristics in the bogie region through STAR-CCM+ based on N$\mathrm{S}$ equations and Lagrangian particle model. By changing the height of apron plate and the inclination angle of front and rear end separation walls, the flow field in bogie region was controlled to prevent and treat of snow accumulation.

In this paper, a flat plate deflector which installed on the middle of bogie frame is proposed to prevent and reduce snow accumulation in the bogie region. Based on Reynolds Averaged Navier-Stokes (RANS) equations combined with the Realizable $k-\varepsilon$ turbulence model and the Lagrangian particle phase, the wind-snow two-phase flow around bogie was simulated by the STAR-CCM+ software. The influences of the deflector with different installation angles on the flow field and the performance of snow accumulation control are studied.

\section{NUMERICAL SET UP}

\subsection{Methodology for CFD Analysis}

The wind-driven snow in high-speed train bogie region is a kind of two-phase flow with continuous and discrete phases. The continuous phase is turbulent airflow around high-speed train, which is simulated by the RANS equations with Realizable $k$ - $\varepsilon$ turbulence model. The $k$ - $\varepsilon$ turbulence model family is widely used with high accuracy and few computational resources in snow drifting simulations (Tominaga et al. 2011a, b; Beyers et al. 2004 a, b; Wang et al. 2019c; Yu et al. 2019.). The continuous phase equation, momentum equation and energy equation can be found in John et al. (1995), $k$ equation and $\varepsilon$ equation in Shih et al. (1995).

In this study, the Lagrangian discrete phase is used to simulate the motion of snow particles. By solving the force balance equations of the particles, the trajectory of the particles in the computational domain can be calculated. The forces acting on one particle include gravity, virtual mass force, shear lift force and pressure gradient force, while the other forces can be ignored. (Liu et al. 2018) The motion equations of the particle are presented as Eq. (1) (Siemens Inc. 2017)

$m \frac{d v_{p}}{d t}=\boldsymbol{F}_{s}+\boldsymbol{F}_{b}$

In Eq. (1), $\boldsymbol{v}_{p}$ denotes the particle velocity, $\boldsymbol{F}_{s}$ represents the forces that act on the surface of the particle, and $\boldsymbol{F}_{b}$ represents the body forces. These forces are decomposed into Eq. (2) and Eq. (3).

$\boldsymbol{F}_{S}=\boldsymbol{F}_{d}+\boldsymbol{F}_{p}+\boldsymbol{F}_{v m}+\boldsymbol{F}_{L S}$

$\boldsymbol{F}_{b}=\boldsymbol{F}_{g}+\boldsymbol{F}_{u}$ 
In Eq. (2) and Eq. (3), $\boldsymbol{F}_{d}, \boldsymbol{F}_{p}, \boldsymbol{F}_{v m}, \boldsymbol{F}_{L S}, \boldsymbol{F}_{g}$ and $\boldsymbol{F}_{u}$ are the drag, pressure gradient force, virtual mass force, shear lift force, gravity force and user-defined body force, respectively. As shown in Table 1. The meanings of the parameters in Table 1 are shown in the Nomenclature.

Table 1 Expression of forces

\begin{tabular}{|c|c|}
\hline Forces & Expression \\
\hline $\boldsymbol{F}_{d}$ & $\boldsymbol{F}_{d}=\frac{1}{2} C_{d} \rho A_{P}\left|\boldsymbol{v}_{s}\right| \boldsymbol{v}_{s}$ \\
\hline $\boldsymbol{F}_{p}$ & $\boldsymbol{F}_{p}=-V_{P} \nabla p_{\text {static }}$ \\
\hline $\boldsymbol{F}_{v m}$ & $\boldsymbol{F}_{v m}=C_{v m} \rho V_{p}\left(\frac{D_{v}}{D_{t}}-\frac{d v_{p}}{d t}\right)$ \\
\hline $\boldsymbol{F}_{L S}$ & $\boldsymbol{F}_{L S}=C_{L S} \frac{\rho \pi}{8} D^{3}\left(v_{S} \times \boldsymbol{\omega}\right)$ \\
\hline $\boldsymbol{F}_{g}$ & $\boldsymbol{F}_{g}=m_{P} \boldsymbol{g}$ \\
\hline $\boldsymbol{F}_{u}$ & $\boldsymbol{F}_{u}=V_{P} \boldsymbol{f}_{u}$ \\
\hline
\end{tabular}

\subsection{Geometry}

This paper aims to study prevention of snow accumulation using the proposed installation of deflector on bogie frame. The geometries of bogie and cavity exert major influences on the flow field of the train bottom (Zhu et al. 2016). However, the realistic geometries of structures in bogie region are extremely complex, such as the enormous nuts, wires and tubes. Therefore, a simplified car body and a bogie are used for numerical simulation in this study. The CRH2 prototype car body is simplified as shown in Fig. 1 with $1.3 \mathrm{~m}$ height and $6.8 \mathrm{~m}$ length. Wang et al. (2018b) have used the same vehicle body model in snow prevention researches.

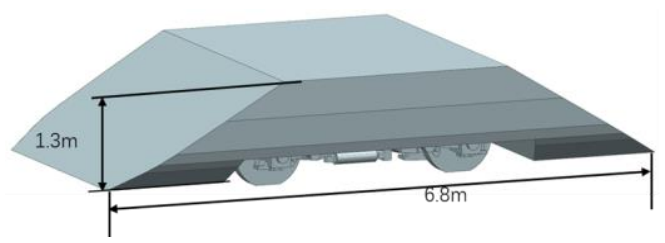

Fig. 1. Simplified vehicle body model.

The important components of CRH2 bogie, such as the brake clamp, gear box and motor are retained in the simplified model as shown in Fig. 2. For the convenience of description, the bogie is divided into front and rear parts according to the flow direction. The numbering of brake clamps is shown in Fig. 2.

According to the literatures (Gao et al. 2018a; Wang et al. 2018b; Xie et al. 2017), particles and airflow move forward smoothly in the early stage of the simulation. When the airflow passes through the middle and rear region of the bogie, the complex geometry of the bogie causes particles to rise with the airflow, and then particles are gradually spread over the bogie region.

In this study, in order to avoid large number of particles entering the bogie region, the idea of installing a flat plate deflector attached on the bottom of the bogie frame is proposed. To satisfy the height limitation of bottom attachments of high-speed train and achieve better flow control, the lowest edges of deflector installed at $90^{\circ}$ are with same height of lowest point of the motor. As a result, the length of the deflector is designed as $2.2 \mathrm{~m}$, the thickness is $0.02 \mathrm{~m}$, and the height is $0.37 \mathrm{~m}$. To study the performance of different installation angles, $30^{\circ}, 60^{\circ}$ and $90^{\circ}$ typical cases are selected for analysis. As shown in Fig. 3(a), case 1 is the original configuration of bogie region without deflector. Case2, case 3 and case 4 are bogies with deflectors at $30^{\circ}, 60^{\circ}$ and $90^{\circ}$ installation angles, as shown in Fig. 3.

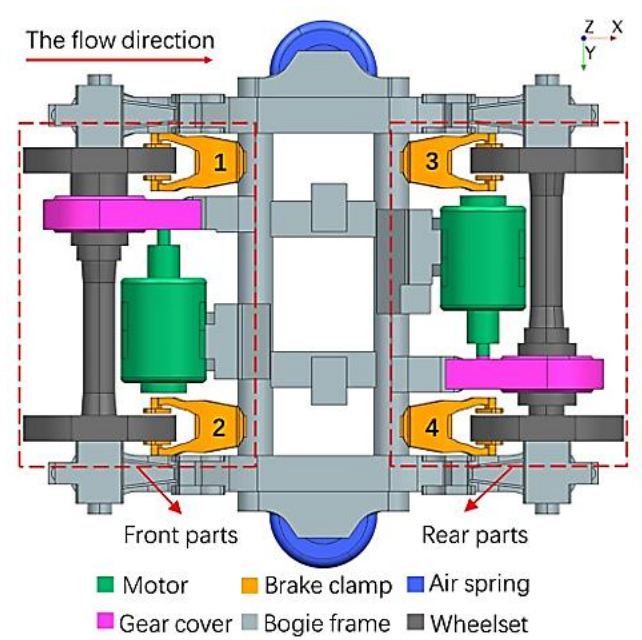

Fig. 2. Simplified bogie model.

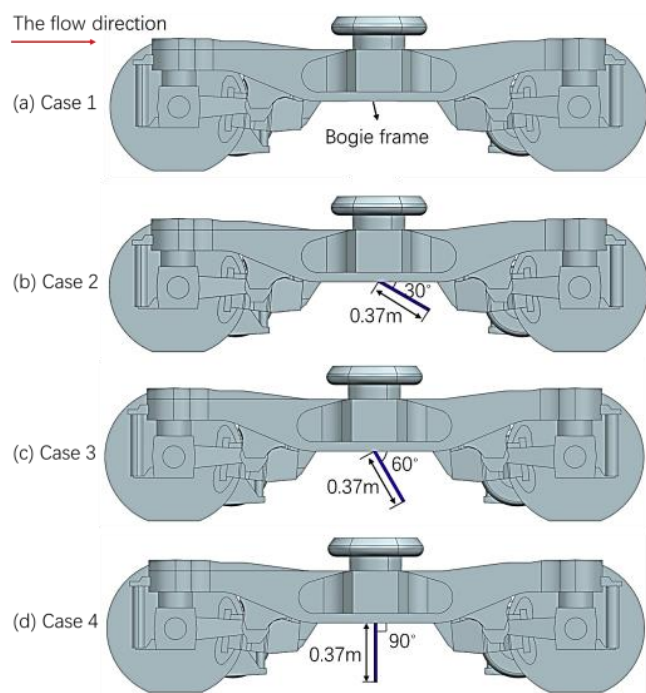

Fig. 3. Installation position of the deflectors.

\subsection{Computational Domain and Boundary Conditions}

The computational domain with dimensions of $43 H \times 20 H \times 10 H$ is illustrated in Fig. 4, where $H$ is the height of the simplified train model which is $1.3 \mathrm{~m}$. The train body and bogie are set as the solid wall surface with no-slip condition. Surface ABFE, ACGE and CDHG are set as the symmetrical plane condition. Surface ABCD is the velocity inlet with $55.56 \mathrm{~m} / \mathrm{s}$, and surface EFGH is the pressure outlet. 
In order to simulate the relative motion between the train and the ground, the surface BDHF and tracks are set as moving wall condition with $55.56 \mathrm{~m} / \mathrm{s}$. For clarity, the boundary conditions are listed in Table 2 .

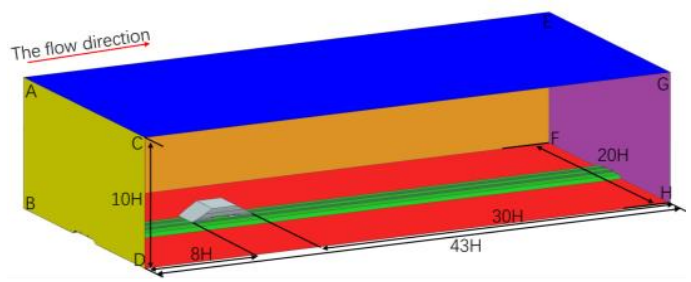

Fig. 4. Computational domain.

Table 2 Details of the boundary conditions

\begin{tabular}{|c|c|c|}
\hline $\begin{array}{c}\text { Domain } \\
\text { boundary }\end{array}$ & $\begin{array}{c}\text { Boundary } \\
\text { condition }\end{array}$ & Value \\
\hline ABCD & Velocity inlet & $55.56 \mathrm{~m} / \mathrm{s}$ \\
\hline EFGH & Pressure outlet & $0 \mathrm{kPa}$ \\
\hline $\begin{array}{c}\text { ABFE、ACGE } \\
\text { CDHG }\end{array}$ & Symmetry plane & $\backslash$ \\
\hline BDHF & Wall & $55.56 \mathrm{~m} / \mathrm{s}$ \\
\hline $\begin{array}{c}\text { Vehicle body } \\
\text { and bogie }\end{array}$ & Wall & 0 \\
\hline
\end{tabular}

The size of the particle emitter is $0.35 \mathrm{~m} \times 2.8 \mathrm{~m}$ which is $1 \mathrm{~m}$ away from the front end of the locomotive, as shown in Fig. 5. According to references (Nemoto et al. 2004; Sang et al. 2012; Liu et al. 2018; Gao et al. $2018 \mathrm{a}$ ), the mass flow rate of snow particles is set as $4 \mathrm{~kg} / \mathrm{s}$, the diameter is $2 \times 10^{-4} \mathrm{~m}$, and the density is $100 \mathrm{~kg} / \mathrm{m}^{3}$. The boundary condition of the particle is set to trap condition. Particle-wall relations on bogie and guide plate are set to trap conditions, train body surfaces are set to reflect conditions and the bottom surface of the calculation domain is set to escape condition.

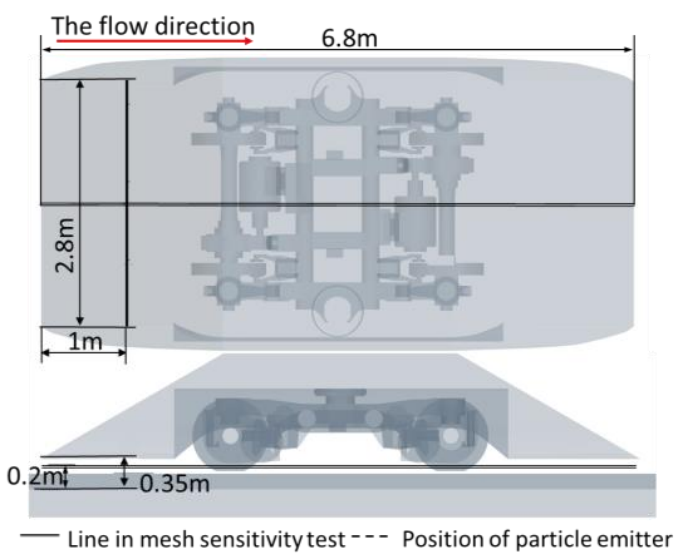

Fig. 5. Line in mesh sensitivity test and position of particle emitter.

In order to ensure the particle motion close to the actual situation, the snow particles are released after $1 \mathrm{~s}$ pre-calculation of pure continuous phase. In order to shorten the calculation time and ensure that particles completely fill the bogie region, the twophase flow simulation time is set as $2 \mathrm{~s}$. Wang et al. (2018c, 2019b) analyzed the changes of snow accumulation on the bogie surface in different simulation times. The results showed that the snow accumulation increased slowly after $2 \mathrm{~s}$.

In this study, the ambient temperature is $-30^{\circ} \mathrm{C}$, and the particle temperature is also $-30^{\circ} \mathrm{C}$, which means no consideration of phase transition or energy release. The dynamic viscosity of air at $-30^{\circ} \mathrm{C}$ is $1.57 \times 10^{-5} \mathrm{~Pa} \cdot \mathrm{s}$ and the density is $1.453 \mathrm{~kg} / \mathrm{m}^{3}$.

\subsection{Grid Generation}

This study uses hexahedral-based hybrid mesh in STAR-CCM+ to mesh the geometric model. The grid size of the bogie surface is set to $0.01 \mathrm{~m}$ and the maximum grid size of the surface of the calculation domain is $2.56 \mathrm{~m}$. Computational domain and bogie surface grid are shown in Fig. 6(a) and Fig. 6(b). The six layers of prism grid refinement with the first layer thickness $0.18 \mathrm{~mm}$ is shown in Fig. 6(c). Thus, the range of $\mathrm{y}^{+}$values is from 30 to 300 , which meets the requirement of chosen turbulence model.

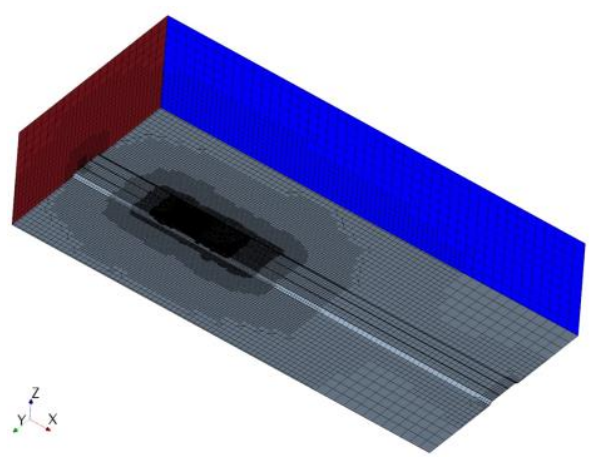

(a)

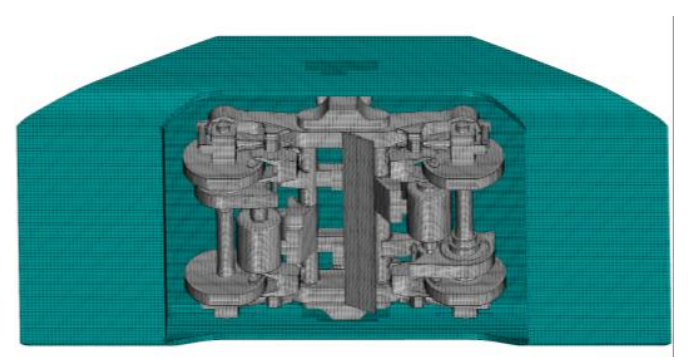

(b)

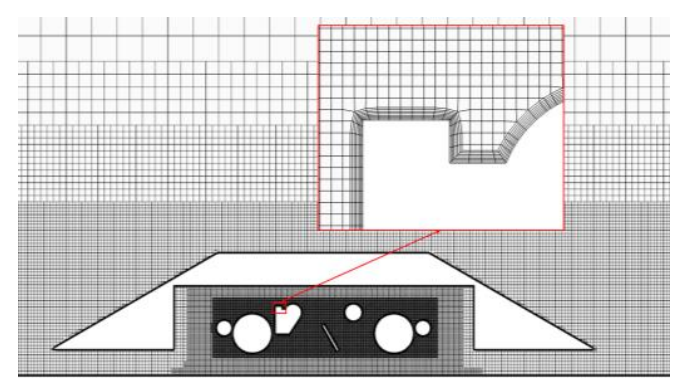

(c)

Fig. 6. Computational mesh: (a) The whole calculation domain; (b) Vehicle body and bogie;(c) Boundary layer. 


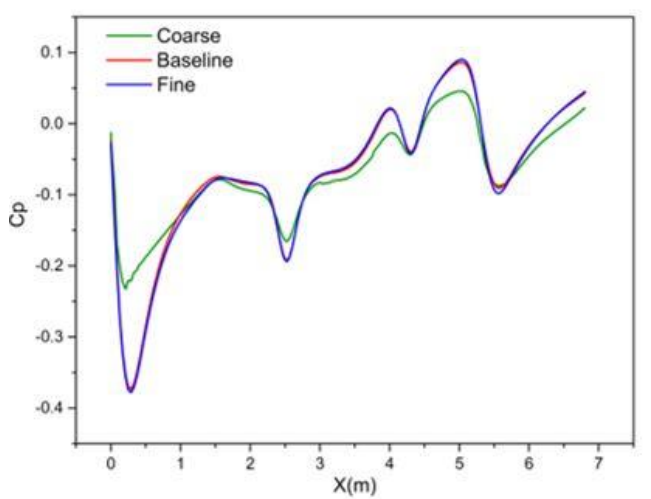

Fig. 7. Comparisons of $C_{p}$ in different grids.

In 3 cases, that the boundary layer refinement methods are the same. The Coarse grids of 8 million is obtained by canceling the encryption around the bogie. The Fine grids of 32 million is obtained by enlarging the encryption area around the bogie. Thus, three sets of grids, Coarse grids of 8 million, Baseline grid of 16 million and Fine grids of 32 million are calculated in mesh sensitivity test.

At the bottom of the train body, a $6.8 \mathrm{~m}$ line segment along the $\mathrm{x}$ direction located at $\mathrm{y}=0$ slice is created to extract dimensionless pressure coefficients, as shown in Fig. 5. The dimensionless pressure coefficients calculated by three sets of grids are compared in Fig. 7. It can be seen that the results of Baseline grid and Fine grid are similar. The result of Coarse grids shows significant difference compared with the two other cases.

In order to further confirm which set of meshes to be used, the masses of snow particles on the bogie surface calculated using Baseline grids and Fine grids were compared, as shown in the Fig. 8. The results show that the difference between the two results was just $0.223 \%$. Considering the simulation efficiency and accuracy, the Baseline grids is used for numerical simulation.

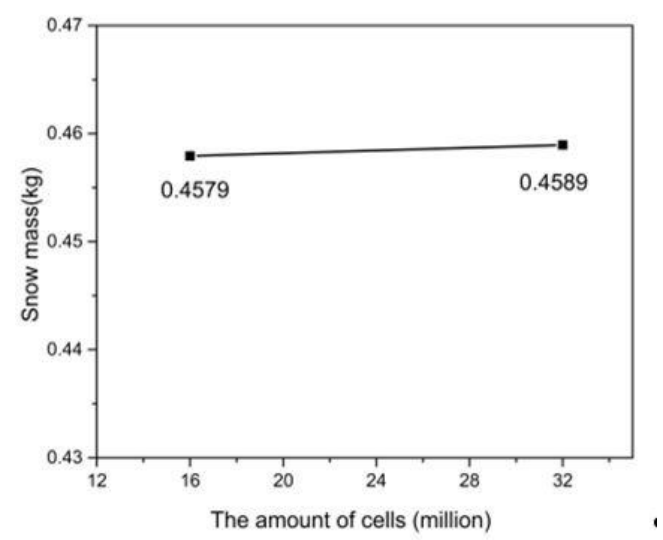

Fig. 8. Comparisons of snow mass in different grids.

\subsection{Numerical Validation}

To verify the accuracy of three-dimensional transient RANS method and Lagrangian particle phase used in the simulation of wind-snow two-phase flow in bogie region, some experiments have already been carried out. Xie et al. (2017) and Wang et al. (2018a, b) have accomplished two-phase wind tunnel tests, in which the pressure and velocity values at specific points are in good agreement with the numerical simulation results. Besides, Gao et al. (2018a) conducted the wind tunnel tests using a 1:2 scaling model to measure the bogie region flow directions with the tuft method which validated the numerical simulation. Besides, the particle distribution experiment of a 1:4 scaling model was carried out with tiny wood chips as replacement of snow particles.

In order to verify the numerical method in this paper, the calculated net wind field and particles accumulation are compared with the experiment results in the literature. The wind tunnel test in Gao et al. (2018a) is numerically reproduced in this paper to compare the thread movements as shown in Fig. 9. It can be seen from Fig. 9(a) that threads NO.4, and 7 indicated the upward air flow. Threads NO.1, $2,3,5,6,9,10,14,15$ and 16 keep almost straight. Threads NO.8, 11, 12 and 13 are moving downward. Figure 9(b) shows that the flow direction is the same as that of the threads indicated in the experiment.

Although the particle accumulation thickness on the surface of bogies cannot be measured in the experiment, the verification of particle phase simulation is achieved by the same snow particle distribution of numerical simulation and two-phase wind tunnel tests. In this paper, the experiment in Gao et al. (2018a) is also simulated to compare the particle accumulation results, as shown in Fig.10. The consistence of the particle distribution and accumulation conditions show that present numerical simulation can accurately simulate the particle distribution on the surface of the bogie.

\subsection{Dimensionless}

For a better comparison in later sections, the velocity and pressure of flow fields and the velocity of particles are normalized as follows:

$$
\begin{aligned}
& U_{x}=u_{x} / U_{r e f} \\
& U_{p x}=u_{p x} / U_{r e f} \\
& C_{p}=\left(P-P_{\infty}\right) /\left(0.5 \rho U_{r e f}^{2}\right)
\end{aligned}
$$

In Eqs. (3), (4) and (5), $u_{x}$ is the time average velocity of $x$ direction, $U_{r e f}$ is the inflow velocity, $u_{p x}$ is the velocity of particle in the $x$ direction, $P$ is the time average pressure, and $P_{\infty}$ is the far field pressure and is set to 0 .

\section{ANALYSIS OF NUMERICAL SIMULATION RESULTS}

\subsection{Streamlines in Bogie Region}

The snow particles are mainly subjected to the gravity and drag force from the high-speed airflow under the train, packing on the bogie surface, thus forming the snow accretion. When the airflow velocity decreases in the up region of bogie, the snow particles can't be blown up according to the smaller 

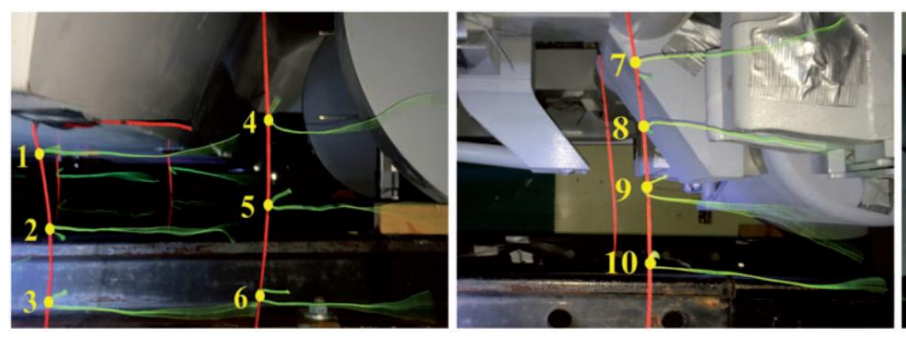

(a)

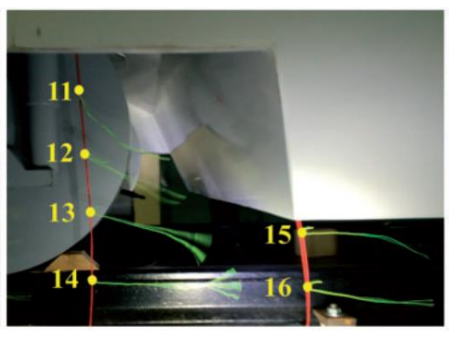

14

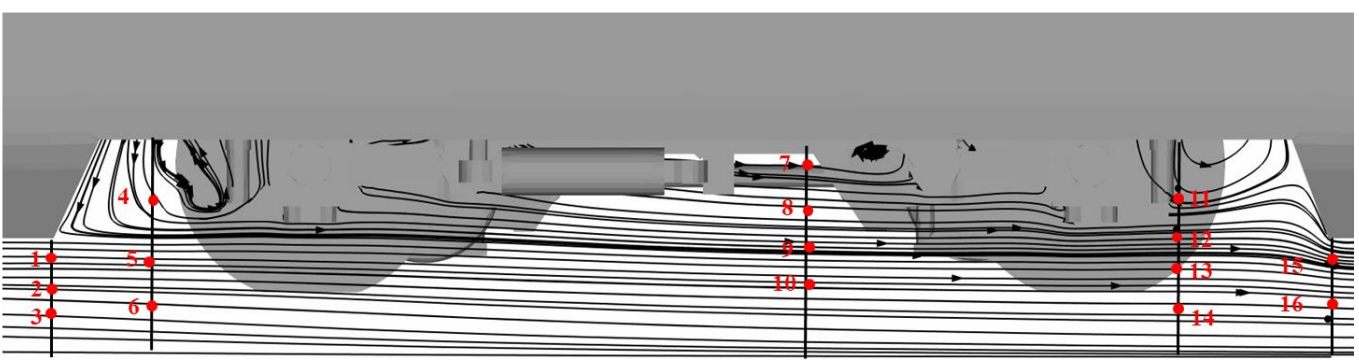

(b)

Fig. 9. Comparison of experiment and numerical simulation:(a) The motion of silk thread in the tunnel experiment; Gao et al. (2018a).(b) Streamlines in the numerical simulation.

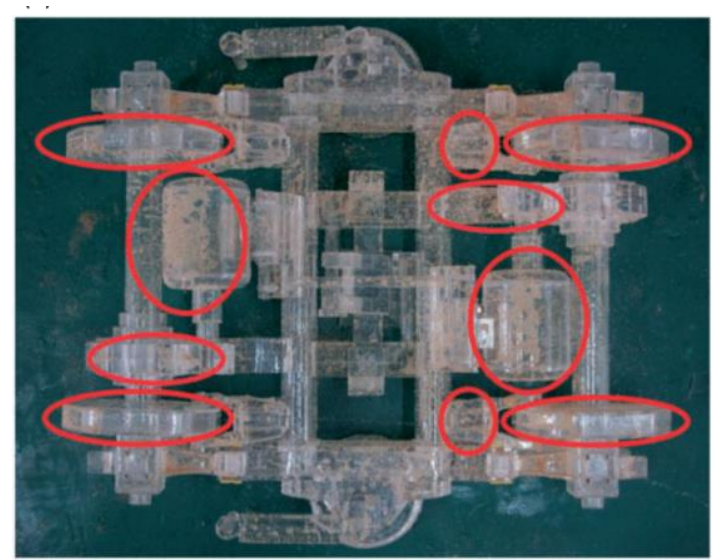

(a)

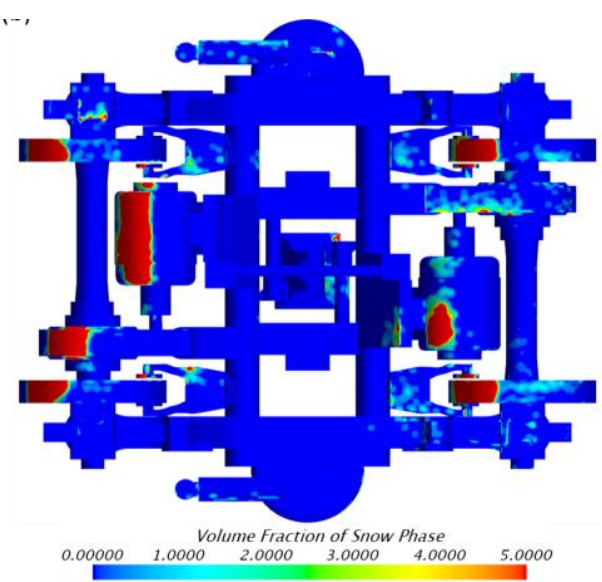

(b)

Fig. 10. Comparison of experiment and numerical simulation: (a) Particle accumulation of tunnel experiment; Gao et al. (2018a). (b) Particle accumulation of numerical simulation.

drag force and will fall onto the surface of the components due to gravity. Therefore, by analyzing the flow field around the bogie region, the motion state of snow particles can be predicted and the reasons for snow accumulation can be uncovered. In this study, two slices are taken for flow field analysis, and their positions are shown in Fig. 11. The streamline colored in $\mathrm{U}_{\mathrm{x}}$ are then shown in Fig. 11, where the positive or negative sign indicate the airflow direction.

Figure 12(a) shows the streamlines of slice1 for the four cases. In comparison with case1, the deflector in case 2 obstructs the movement of high-speed airflow from entering the upper area. Therefore, the number of snow particles entering the upper part of the bogie is reduced. In case 3 , the deflector diverts most of the high-speed airflow to region under the bogie, which greatly reduces the scouring of snow particles on the parts in rear bogie cavity. Therefore, fewer snow particles pile up on the surface of the rear bogie components in case 3 compared to case1. The deflector with $90^{\circ}$ almost divides the airflow into two parts. One part enters the front region of the bogie and the other part enters the rear side along the deflector. Therefore, comparing with case1, snow accumulation on the frame of case 4 is thicker.

As shown in Fig. 12(b), the streamlines of the four cases at the location of slice 2 are compared. For the rear side brake clamps, the installation of the deflector greatly lightens the scouring of high-speed airflow on this part. Therefore, snow particles accumulated on the surface of the rear side brake clamps will be fewer in cases with the flat plate deflector compared with case1. In case2, the 


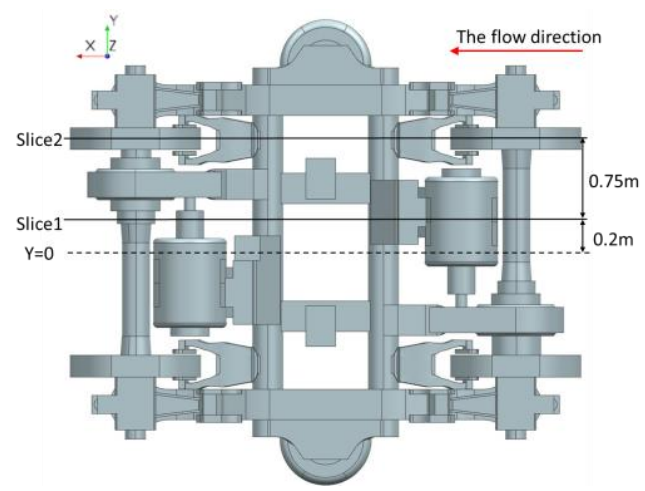

Fig. 11. Positions of slices in the bogie region.
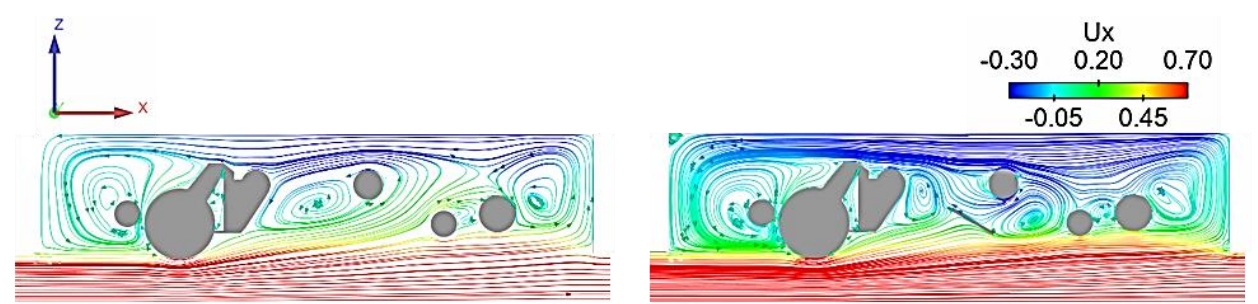

Case1

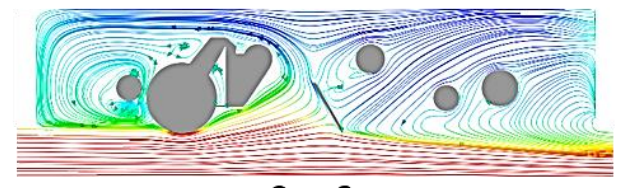

Case2

Case3

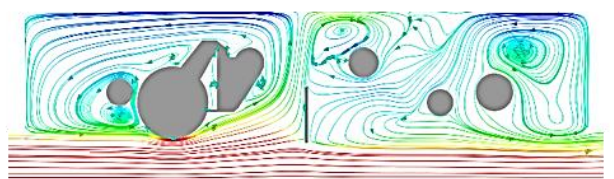

Case4

(a)

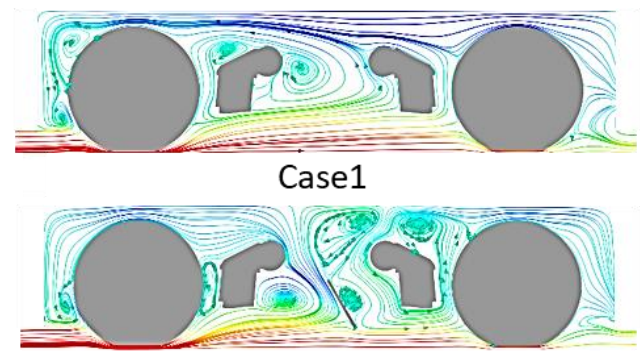

Case3

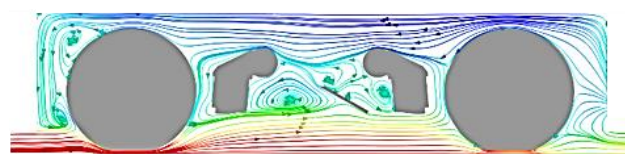

Case2

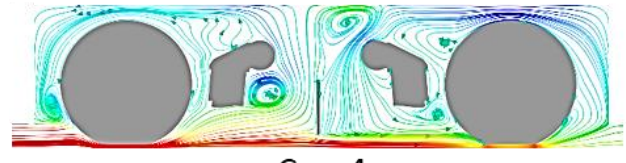

Case4

(b)

Fig. 12. Streamlines in the bogie region: (a) slice1; (b) slice2.

deflector causes airflow to circulate which forms a low-speed vortex under the lower part of the brake clamps. Therefore, snow on the front brake clamps may be thicker than case 1 . In case3, although a small part of the airflow directly scours the front brake clamp surface due to the deflector installed at $60^{\circ}$, the airflow entering the upper space of the bogie has a weak velocity to carry snow particles. This means that the overall number of particles settled on the upper space of the bogie in case 3 may be fewer than case1. Similarly, in case4, the velocity of airflow above the bogie is lower which lead to fewer particles accumulated on the upper surface of bogie. However, it is worth to notice that airflow along the rear side of the deflector goes up to the upper region. Therefore, there may be an uneven distribution of snow particles in the front and rear side of the bogie in case4.

\subsection{Pressure Distributions Around the Bogie}

Figure 13 shows the pressure coefficient distribution of each section. The pressure coefficient at the rear of bogie decreases in value and area under the influence of deflector, which indicates the reduction of snow particles entering the bogie region. Meanwhile, the positive pressure on the surface of the rear motor in cases with deflector is much smaller than original case. That means the deflector protects component s such as the motor from the scour of high-speed airflow. It can be drawn from Fig. 13(b) 


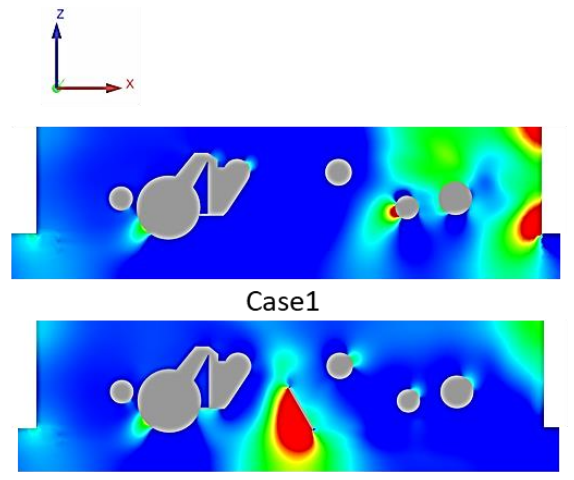

Case3

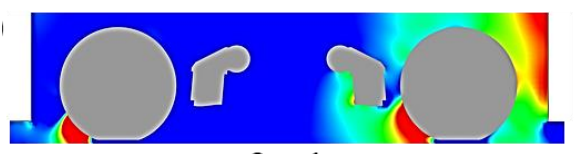

Case1

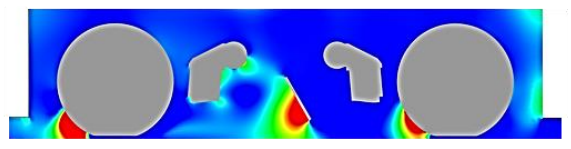

Case3

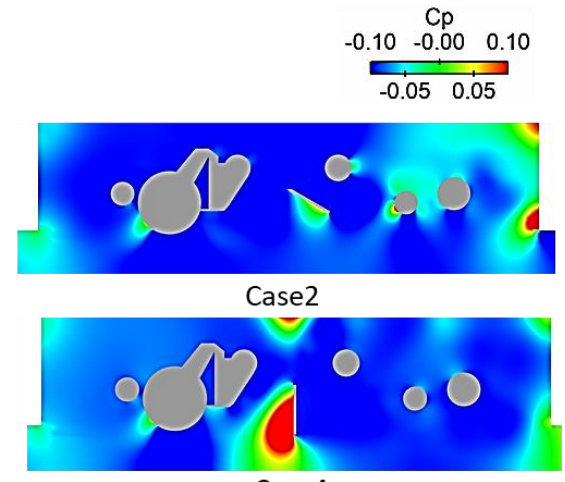

Case4

(a)

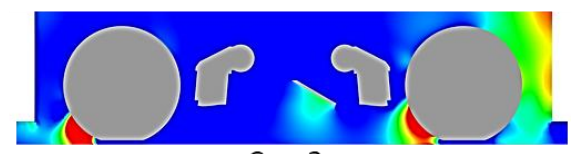

Case2

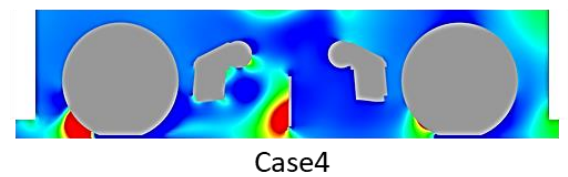

(b) that, under the effect of the deflector, the negative pressure on the front side of the brake clamps in case 3 and case 4 is smaller than case 1 . In addition, the surface of the rear brake clamp are subjected to negative pressure, and the range of positive pressure on the surface of the rear wheelset decreases. As a result, deflectors deliver protections for the rear parts.

\subsection{Particle Distributions in Bogie Region}

As shown in Fig. 14, the distribution state of particles in the bogie at $2 \mathrm{~s}$ is colored with $U_{p x}$ for the four cases where positive or negative sign indicates the direction of particle motion. Snow particles move upward into the front of bogie region, as is shown in Fig. 14(a). Some particles follow the airflow at the rear end bogie cavity to enter the upper region of the bogie region and flow back to the front half. As a consequence, the upper part of the bogie cavity is filled with low-speed particles. As shown in Fig. 14(b) for case2, the deflector installed at $30^{\circ}$ inhibits the ascending particles. But relatively small attack angle of deflector can avoid some particles ascending with airflow at the rear end of the bogie cavity then entering the upper part of the bogie. For case3, deflector with $60^{\circ}$ attack angle has a relatively obvious effect on prevention of particles ascending at the rear end of bogie cavity, seeing Fig. 14(c). However, a small percentage of particles will follow the airflow rebounded in front of deflector to fly into the front region of the bogie cavity. In Fig 14(d), the vertically installed deflector in case 4 maximally redirected the snow particles. However, the particles will follow the airflow along the deflector, then enter the rear of bogie region resulting in a significant decrease of snow particles distribution in the front region but an increase of snow particles distribution in the rear region.

\subsection{Particle Accumulation on Bogie Surface}

Figure 15 shows the snow thickness on the surface of the bogie frame and auxiliary components from bottom view and top view under four cases. In case1, snow particles are accumulated on the bottom surface as well as on the top, as shown in Fig. 15(a). And the particles accumulated on the surface of rear parts are more than the front parts. The snow particles also pile up at the bottom of the four brake clamps. A large amount of snow particles can also be seen on the upper surface of the rear motor. In addition, the snow particles accumulated on the surface of the bogie frame is serious. In case2, the snow accumulation on the top and bottom surface is reduced obviously, seeing Fig. 15(b). This reduction is more pronounced in the rear parts. However, snow accumulation on the air springs increases slightly. In case3, the snow packing on bogie is significantly less compared with case1. The snow accumulation on the surface of the rear brake clamps and rear gear boxes is significantly improved. In case4, snow particles accumulated slightly fewer on the bottom surface but more to a certain extent on the top surface compared with case1, especially at the bogie frame crossbeam. Comparison among cases 2,3 and 4 shows that the performance of snow accumulation prevention in case 2 and case 3 may be better. In case3, the snow accumulation on the motor surface is more serious than that on case2, which may 


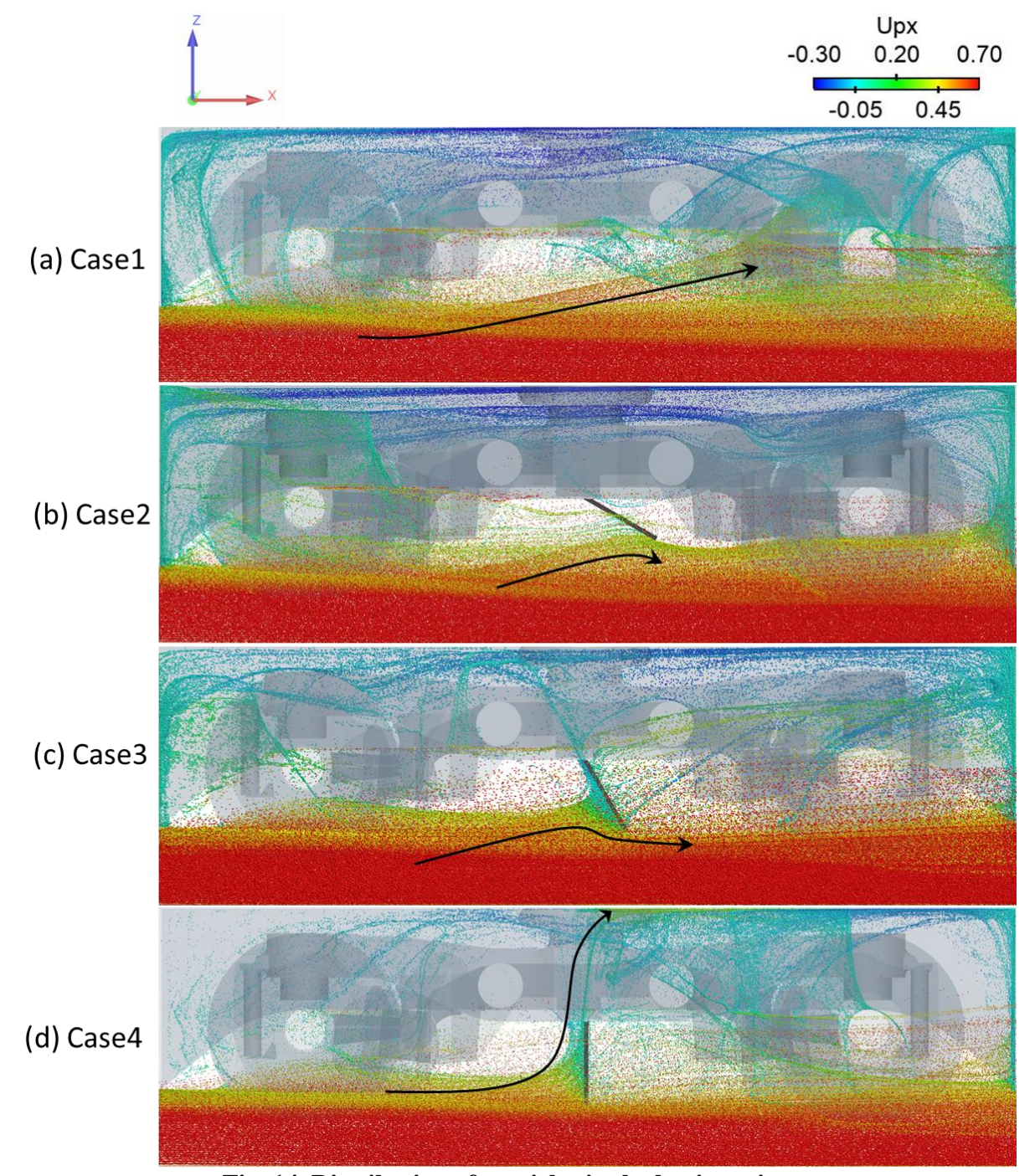

Fig. 14. Distribution of particles in the bogie region.

indicate that the performance of the deflector in case 2 is better than that in case3. However, snow accumulation on the surface of many front parts and top surface of rear parts in case 3 is less than that in case2. Therefore, the performance of the deflector in case 2 and case 3 can only be obtained through further quantitative analysis. Overall, the conclusions of above qualitative analysis are consistent with the results of flow field and particle motion.

Figure 16 presents the snow mass on the bogie surface in different cases at $2 \mathrm{~s}$. Owing to the snow mass on the surface of different parts varies a lot, two subplots, Fig.16 (a) and (b) are provided for clearly comparison. It can be seen from Fig.16(a) and (b) that, compared with case1, the snow packing on the frame surface in case 2 and case 3 is obviously reduced, while the snow packing on the frame surface in case 4 is slightly increased. In the cases with the deflectors, the snow mass of the front gear box and the front motor increase less and the snow mass of the brake clamp2 increases more.
Fortunately, under the influence of the deflectors, the snow mass on the surface of other components is reduced.

Overall, the results of quantitative analysis of snow mass on the surface of components are consistent with those of qualitative analysis of snow accumulation. In addition, it can be seen that the accumulated snow on the surface of the rear gear box, the rear motor and the rear brake clamp is significantly reduced after the addition of the deflector It shows that the deflector plays a key role in protecting the gear box and the motor on the rear side of the bogie region.

\subsection{Particle Change Rate on Bogie Surface}

For a more straightforward comparison of anti-snow performance in different cases, the snow accumulation change rate of cases with deflector based on case 1 are provided in Table 3. The change rate of snow accumulation mass on the surface of each component is also shown in Fig. 17. 
(a) case1

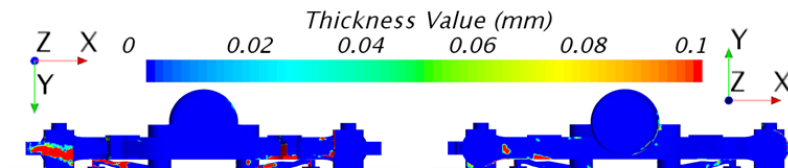

(b) case2

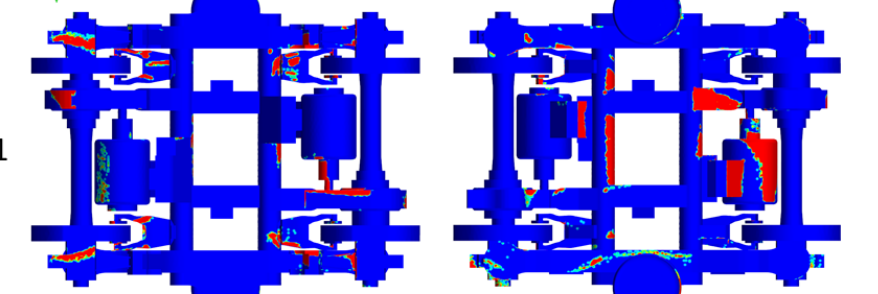

(c) case3

(d) case4

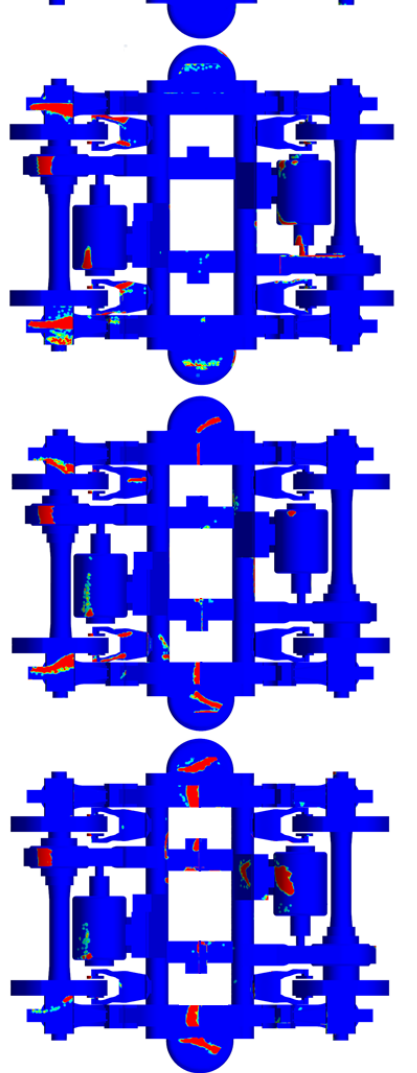

Bottom surface

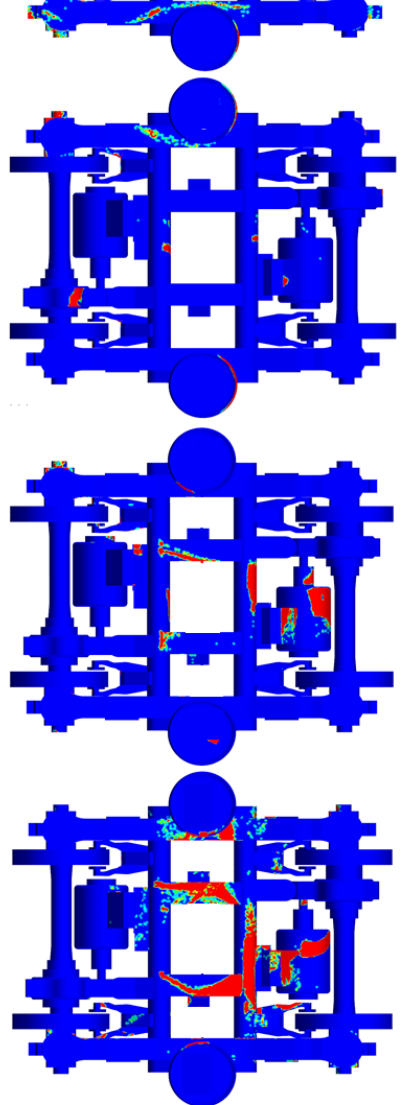

Top surface

Fig. 15. Snow accumulation on the bogie.

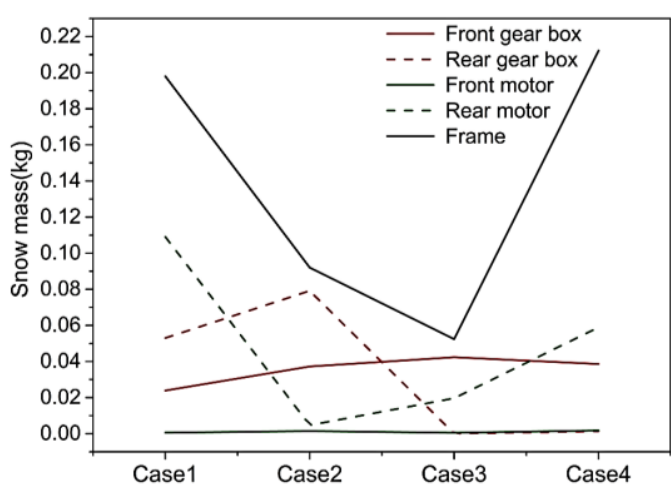

(a)

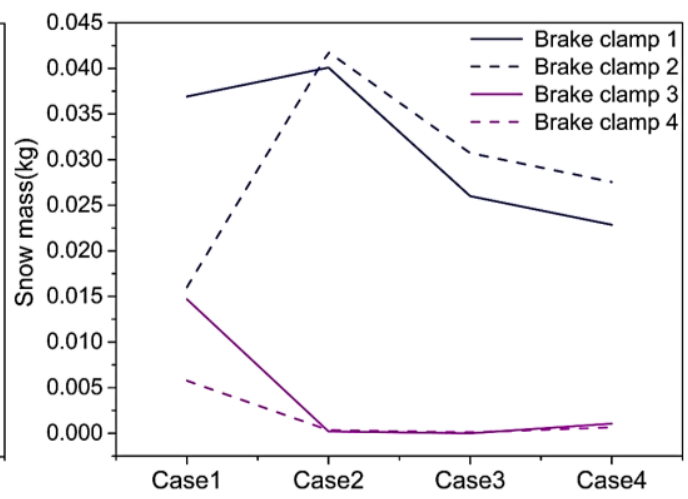

(b)

Fig. 16. Snow accumulation mass of each bogie component for 4 cases. 
G. Gao et al. / JAFM, Vol. 13, No. 5, pp. 1377-1389, 2020.

Table 3 The snow accumulation change rate of three cases with deflector

\begin{tabular}{|c|c|c|c|}
\hline Parts & Case2 & Case3 & Case4 \\
\hline Front gear box & $55.80 \%$ & $77.27 \%$ & $61.64 \%$ \\
\hline Rear gear box & $49.41 \%$ & $-99.81 \%$ & $-97.47 \%$ \\
\hline Front motor & $150.30 \%$ & $-4.99 \%$ & $196.97 \%$ \\
\hline Rear motor & $-95.68 \%$ & $-81.99 \%$ & $-45.95 \%$ \\
\hline Brake clamp 1 & $8.56 \%$ & $-29.60 \%$ & $-38.10 \%$ \\
\hline Brake clamp 2 & $160.80 \%$ & $91.82 \%$ & $71.98 \%$ \\
\hline Brake clamp 3 & $-98.56 \%$ & $-99.99 \%$ & $-92.73 \%$ \\
\hline Brake clamp 4 & $-94.00 \%$ & $-98.26 \%$ & $-88.47 \%$ \\
\hline Frame & $-53.52 \%$ & $-73.54 \%$ & $7.16 \%$ \\
\hline Sum & $-35.14 \%$ & $-62.46 \%$ & $-20.30 \%$ \\
\hline
\end{tabular}

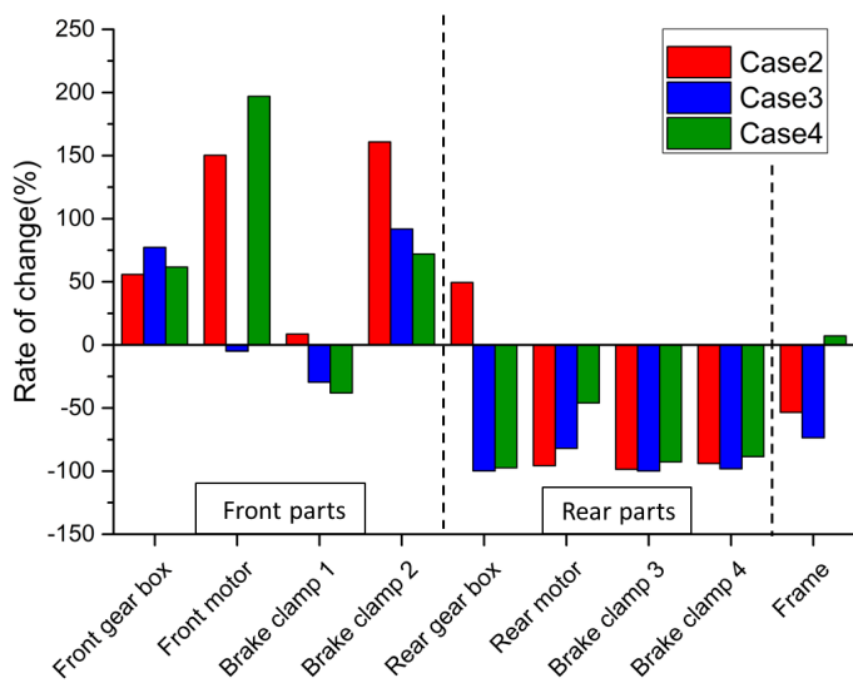

Fig. 17. Change rate of snow accumulation mass on each component surface in case2, case3, and case4.

It can be inferred that snow packing of most components on the rear part of the bogie is reduced after the installation of the flat plate deflector. This indicates that the deflectors play a positive role in the flow field at the rear of the bogie, reducing the erosion of snow particles on the parts at the rear. Thus, the snow accumulation at the rear of the bogie is lessened and the effect of snow prevention is achieved. By observing the snow accumulation on the parts in the front half of bogie cavity, although snow accumulation on some parts is diminished, the mass of snow on more parts increases. It is worth to notice that, although the flat plate deflector performs unsatisfied on the front gear box and the front motor respect to the snow change rate, the actual added snow of the other three cases is very small in mass, as shown in Fig. 16(a). Hence, deflector installed under bogie frame can be concluded with almost no influences on the snow accumulation on parts in the front half of bogie region but largely improved the snow accumulation on parts in the rear half of bogie region.

To be more straightforward, the change rate of snow on front parts, rear parts, frame and whole bogie are presented in Fig. 18. Obviously, all three installation angles of deflectors can alleviate the snow accumulation problem in the bogie region, among which the flat plate deflector installed at $60^{\circ}$ has the best effect. However, the best installation angle of deflector is still need to be investigated.

\section{Conclusions}

In this paper, the anti-snow performances of the deflector with three installation angles have been numerically studied using wind-snow two-phase flow simulations. The influences of the deflector on the flow field and snow accumulation in the bogie region are summarized as follows:

1) The flat plate deflector can change the movement and distribution state of the particles in the bogie region. However, the influence of the deflector with different installation angles on the particle accumulation on the bogie components are different. The deflector installed at $30^{\circ}$ prevents a small 


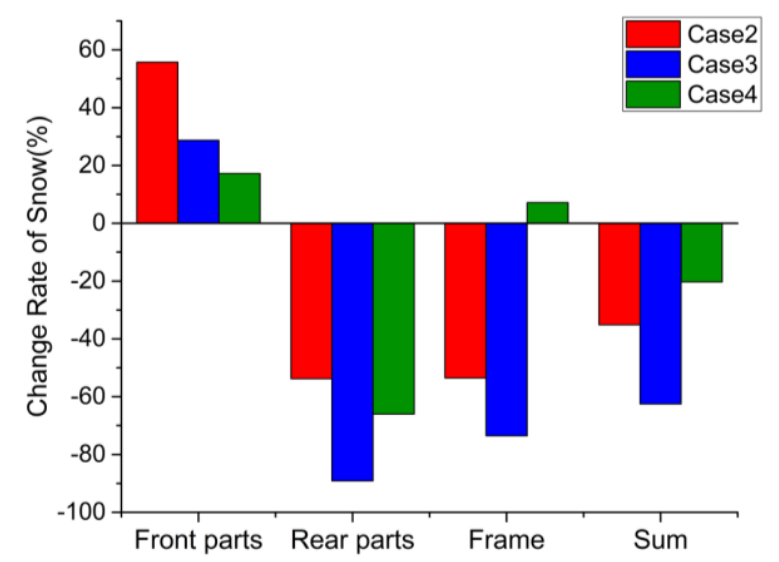

Fig. 18. Change rate of snow accumulation on different parts in case2, case 3 and case4.

number of snow particles from moving up the rear end of the bogie chamber with the airflow. The deflector installed at $60^{\circ}$ may have a relatively better performance than deflector at $30^{\circ}$, which causes most of the particles pass through bogie region underneath the bogie frame. Besides, the deflector installed at $90^{\circ}$ resulting in a significant reduction of snow particles distribution in the front region but an increased distribution in the rear region.

2) The flat plate deflector will change the number of particles entering the bogie region and affect the mass of snow on the surface of bogie components. Overall, all three angles of deflectors can reduce the mass of snow accumulated in the bogie region. The deflector installed at $60^{\circ}$ has the best effect with $62.46 \%$ reduction in snow accumulation. The deflector installed at $30^{\circ}$ works out with $35.14 \%$ reduction. The deflector installed at $90^{\circ}$ has the smallest snow accumulation reduction of $20.30 \%$. It is worth to notice that no matter what angle the deflector is, the snow accumulated on components in the rear side is reduced.

In this study, only the installation angle is discussed for proposed deflector, without considering the influences of height and thickness of deflector on wind-snow flow. However, the study of installation angle was also limited on only three typical degrees $\left(30^{\circ}, 60^{\circ}\right.$ and $\left.90^{\circ}\right)$. Besides, this paper only studies the snow prevention effect, without considerations of deflector on train resistance, running stability or other aspects. In the actual situation, snow is also accumulated on the surface of the deflector. In-depth discussions of above questions are worth to be launched in the future researches.

\section{ACKNOWLEDGEMENTS}

The authors acknowledge the computing resources provided by the High-speed Train Research Centre of Central South University, China.

\section{FUNDING}

This work is supported by the National Key Research and Development Program of China [Grant No. 2016YFB1200404], the National Science Fund Youth Science Fund of China [Grant No. 51605044 and U1534210], and the Science Foundation of Hunan Province [Grant No. 2016 ji3004 and No.2019JJ50790].

\section{REFERENCES}

Bettez, M. (2011). Winter technologies for high speed rail. Norwegian University of Science and Technology, Trondheim, Norway.

Beyers, J., P. A. Sundsbo and T. M. Harms (2004b) Numerical simulation of three-dimensional, transient snow drifting around a cube. Journal of Wind Engineering and Industrial 92(9), 725747.

Beyers, J. H. M. (2004a) Numerical modeling of snow flow characteristics surrounding SANAE IV Research Station. Ph. D. thesis. University of Stellenbosch, Stellebosch, South Africa.

Cai, H. (2018). Research on anti-snow in the bogie region of high-speed train using shape optimization. MSc thesis. Southwest Jiaotong University, Chengdu, China.

Fujii, T., K. Kawashima and S. Iikura (2002). Preventive measures against snow for highspeed train operation in Japan. Cold Regions Engineering Cold Regions Impacts on Transportation and Infrastructure 448-459.

Gao, G., Y. Zhang and F. Xie (2018a). Numerical study on the anti-snow performance of deflectors in the bogie region of a high-speed train using the discrete phase model. Proceedings of the Institution of Mechanical Engineers, Part F: Journal of Rail and Rapid Transit 233(2), 141-159.

Gao, G., Y. Zhang and J. Zhang (2018b). Effect of 
bogie fairings on the snow reduction of a highspeed train bogie under crosswinds using a discrete phase method. Wind and Structure 27(4), 255-267.

John, D. and Anderson, J. R. (1995). Computational Fluid Dynamics: The Basics with Applications. McGraw-Hill Series in Mechanical Engineering.

Kloow, L. (2006). High-speed train operation in winter climate. Transrail Publication BVF5.

Liu, M., J. Wang and H. Zhu (2018). A numerical study of snow accumulation on the bogies of high-speed trains based on coupling improved delayed detached eddy simulation and discrete phase model. Proceedings of the Institution of Mechanical Engineers, Part F: Journal of Rail and Rapid Transit 233(7), 715-730.

Nemoto, M. and K. Nishimura (2004) Numerical simulation of snow saltation and suspension in a turbulent boundary layer. Journal of Geophysical Research Atmospheres 109(D18).

Paradot, N., E. Allain and X. De La Casa (2014, June). A numerical modelling of the snow accumulation on a high-speed train. Frist International conference in numerical and experimental aerodynamics of road vehicles and trains (Aerodynamics 1), Bordeaux, France, 23-25.

Sang, J. (2012). Numerical simulation of blowing snow with mixed diameter. Lanzhou University, Lanzhou, China.

Shih, T.H., W.W. Liou and A. Shabbir (1995). A new $k-\varepsilon$ eddy viscosity model for high Reynolds number turbulent flows. Comput. Fluids 24(3), 227-238.

Siemens Inc, STAR-CCM-user's guide (2017).

Tominaga Y., T. Okaze and A. Mochida (2011b). CFD modeling of snowdrift around a building: An overview of models and evaluation of a new approach. Building and Environment 46(4), 899-910.

Tominaga, Y., A. Mochida and T. Okaze (2011a). Development of a system for predicting snow distribution in built-up environments: Combining a mesoscale meteorological model and a CFD model. Journal of Wind Engineering and Industrial Aerodynamics 99(4),460-468.

Wang, J., G. Gao and M. Liu (2018a). Numerical study of snow accumulation on the bogies of a high-speed train using URANS coupled with discrete phase model. Journal of Wind Engineering and Industrial Aerodynamics 183: 295-314.

Wang, J., G. Gao and Y. Zhang (2019b). Anti-snow performance of snow shields designed for brake calipers of a high-speed train. Proceedings of the Institution of Mechanical Engineers, Part F: Journal of Rail and Rapid Transit 233(2): 121140.

Wang, J., H. Liu and Z. Chen (2019c). Probabilitybased modeling and wind tunnel test of snow distribution on a stepped flat roof. Cold Regions Science and Technology 163: 98-107.

Wang, J., J. Zhang and F. Xie (2018b). A study of snow accumulating on the bogie and the effects of deflectors on the de-icing performance in the bogie region of a high-speed train. Cold Regions Science and Technology 148:121-130.

Wang, J., J. Zhang and Y. Zhang (2018c). Impact of bogie cavity shapes and operational environment on snow accumulating on the bogies of high-speed trains. Journal of Wind Engineering and Industrial Aerodynamics 176: 211-224.

Wang, J., J. Zhang and Y. Zhang (2019). Impact of rotation of wheels and bogie cavity shapes on snow accumulating on the bogies of high-speed trains. Cold Regions Science and Technology 159: 58-70.

Wiebe, E., L. F. (2010, October 20). Factsheet Snow Deflector. from UIC: http://www.uic.org/forms/IMG/pdf/005_factsh eet_snow_defelctor.pdf

$\mathrm{Wu}, \mathrm{Y}$. (2015). Design and research of simulation the high-speed rail specific location devine of the snowproof. MSc thesis. Hunan University of Technology, Zhuzhou, China.

Xie, F., J. Zhang and G. Gao (2017). Study of Snow Accumulation on a High-Speed Train's Bogies Based on the Discrete Phase Model. Journal of Applied Fluid Mechanics 10(6), 1729-1745.

Yu, Z., F. Zhu and R. Cao (2019). Wind tunnel tests and CFD simulations for snow redistribution on 3D stepped flat roofs. Wind and Structures, 2019, 28(1): 31-47.

Zhu, J., Z. Hu and D. J. Thompson (2016). Flow behaviour and aeroacoustic characteristics of a simplified high-speed train bogie. Proceedings of the Institution of Mechanical Engineers, Part F: Journal of Rail and Rapid Transit 230(7): 1642-1658. 